\title{
Health-Related Quality of Life for People with Type 2 Diabetes in Saudi Arabia: A Cross-Sectional Study
}

Mohammed J. Alramadan ( $\nabla$ mohammed.alramadan@monash.edu )

Monash University Faculty of Medicine Nursing and Health Sciences https://orcid.org/0000-0002-4544-9579

Afsana Afroz

Department of Epidemiology and Preventive Medicine, Monash University, Melbourne

Bodrun N. Siddiquea

Department of Epidemiology and Preventive Medicine, Monash University, Melbourne

Noor A. Alabdullah

AL-Luluah Dental Complex, Mecca

Sara M. Alameer

King Khalid University Hospital - King Saud University Medical City, Riyadh

Adel Mehmas $\mathrm{H}$. Alragas

Medical City - King Saud University, Riyadh

Ghada Mohammed Amin

Medical City - King Saud University, Riyadh

Nour A. Bayaseh

University of Science and Technology, Sana'a

Fatimah A Alramadan

Hofuf

Baki Billah

Department of Epidemiology and Preventive Medicine, Monash University, Melbourne

Research

Keywords: Type 2 diabetes, health related quality of life, HRQoL, Saudi Arabia

Posted Date: December 30th, 2019

DOI: https://doi.org/10.21203/rs.2.19689/v1

License: (c) (i) This work is licensed under a Creative Commons Attribution 4.0 International License. Read Full License 


\section{Abstract}

Background: The aim of the study was to assess the health-related quality of life and its associated factors among people with type 2 diabetes in Saudi Arabia.

Methods: A cross-sectional study was conducted among adults with type 2 diabetes who attended diabetes centres in three major cities in Saudi Arabia in 2017. Participants were interviewed and their medical records were reviewed. The EuroQol 5-Dimension 5-Level was used to assess health-related quality of life, and bootstrap resampling with multiple regression analysis was used to identify its associated factors.

Results: A total of 1121 participants were recruited. The mean age was $57.6 \pm 11.1$ years, and the mean utility index of the quality of life was $0.739 \pm 0.261$ ( $0.795 \pm 0.197$ for males and $0.709 \pm 0.285$ for females) and dropped to $0.566 \pm 0.339$ in the presence of micro- and macro-vascular complications. Among the study participants, $51.0 \%, 12.3 \%$, and $30.5 \%$ had problems with mobility, self-care and usual activity, respectively, while $51.6 \%$ and $45.8 \%$ had problems with pain-discomfort and anxiety-depression, respectively. Factors associated with low health-related quality of life were low education level, physical activity $<150$ minutes per week, long sitting time, anxiety, depression and diabetes complications.

Conclusions: Health-related quality of life is low among people with type 2 diabetes in Saudi Arabia, particularly among women and those who had diabetes complications. In order to improve the quality of life, people with type 2 diabetes should maintain a healthy lifestyle, and appropriate interventions should be taken to prevent complications and comorbidities.

\section{Introduction}

Globally, diabetes is a major and continuously growing health issue. This chronic disease is the most common endocrine disorder, affecting $8.8 \%$ of the adult population worldwide, and it is projected that the global prevalence of the disease will reach $9.9 \%$ by 2045 [1]. Diabetes occurs when there is a defect in insulin secretion, insulin action or both, which results in chronically elevated blood glucose level [2]. A high blood glucose level can damage large and small blood vessels, leading to multiple diabetes complications, disability and reduced life expectancy. There are three major types of diabetes: type 1 , type 2 and gestational. Type 2 diabetes mellitus (T2DM), which mainly affect adults, is the most common type, accounting for $90 \%$ of cases of diabetes [2]. Unhealthy eating habit, physical inactivity and obesity are the main risk factors for T2DM [2].

T2DM is managed by lifestyle modification and medication to control blood glucose level. The main aim of the management is to maintain health and quality of life of the affected people so that they live a normal life similar to those without diabetes. Health-related quality of life (HRQoL) focuses on a person's perception of wellbeing, including their physical and mental health [3], and is considered a multidimensional concept that involves the evaluation of the positive and negative aspects of a person's life [4]. Currently, HRQoL is recognised as an important tool in the assessment of the effect of chronic diseases on quality of life, and is an important addition to the traditional measures of clinical outcomes, such as morbidity and mortality [5]. HRQoL also allows policy makers to better understand the burden of chronic diseases and can be useful for evaluating the cost effectiveness of health programs and interventions [6]. Various generic and disease-specific tools that assess HRQoL have been used for people with diabetes [7]. Among these tools the EuroQol 5-Dimension is the most widely used tool because of its simplicity, reliability and its ability to be used in cost-effectiveness analyses $[5,8]$.

Maintaining a good HRQoL for people with diabetes is challenging for both the affected persons and the healthcare systems. Over the long-term, diabetes can lead to complications that can have an adverse effect on health and HRQoL. Many studies have shown that people with diabetes have lower HRQoL compared to people without diabetes [9-11], especially when they have diabetes-related complications [9, 12, 13]. Studies also showed that age, gender, socioeconomic status, obesity, duration of diabetes, hyperglycaemia, hypoglycaemia, insulin use, and the use of multiple medications were associated with HRQoL [12-16]. Furthermore, the mere awareness of having diabetes had been found to impact the HRQoL [17]. A person's beliefs, experiences and expectations can also affect HRQoL and influence how other factors can affect it. This means that factors that affect HRQoL may vary between different populations. Thus, identifying factors that affect HRQoL in each individual population is an essential step towards controlling these factors and maintaining quality of life for people with diabetes.

The Gulf Cooperation Council (GCC) countries, including Saudi Arabia, Kuwait, Bahrain, Qatar, the United Arab Emirates and Oman have witnessed vast economic and lifestyle changes over the past few decades. Along with these changes there has been a rapid increase in the prevalence of diabetes mellitus. Currently, Saudi Arabia and the other GCC countries are among those with the highest prevalence of the disease, both regionally and globally [2]. Compared to the global prevalence (8.8\%) and the regional prevalence (10.7\%) of diabetes among adults, Saudi Arabia has a considerably higher prevalence of 17.7\% [2]. Previous studies have also shown that the prevalence of diabetes complications was high among Saudis with diabetes [18, 19] and that their HRQoL was low $[11,20]$.

A few studies have assessed HRQoL among people with T2DM in Saudi Arabia. These studies were limited to small samples and small geographical locations [11, 20-22]. In addition, it is challenging to compare the results of these studies due to the use of different tools in the assessment of HRQoL. Further, factors associated with overall HRQoL and each of its dimensions have not been explored adequately. The aim of this study was to assess the HRQoL among people with T2DM in Saudi Arabia and to explore the effect of various demographic, behavioural and clinical factors on HRQL and each of its dimensions.

\section{Methods}

\section{Study Design and Population}


The study was conducted as a cross-sectional survey. The study population consisted of attendees of diabetes centres in the cities Hofuf, Riyadh, and Jeddah. Inclusion criteria were age 18 years or older, documented diagnosis of T2DM, and duration of diabetes of at least one year. Pregnant women and people with other types of diabetes, including type 1 and gestational, were excluded from the study. The target was to recruit 1082 participants, which was based on a sample size calculation with a $90 \%$ confidence level, $5 \%$ significance level, $2.5 \%$ margin of error around the previously reported prevalence of diabetes complications and comorbidities in Saudi Arabia [23]. Ethical approval was obtained from the Monash University Human Research Ethics Committee in Australia and the Research Ethics Committee of the Ministry of Health in Saudi Arabia. All study procedures were carried out in accordance with the principles of the Declaration of Helsinki as revised in 2013.

\section{Data collection}

Data collection took place between the 15th of May and the 30th of November 2017. Trained data collectors randomly approached consecutive attendees of the diabetic centres and explained the study to them. Written informed consent was obtained upon their approval to participate, then they were interviewed face-to-face using a structured, pre-tested questionnaire [23]. The collected information included socio-demographics, behavioural and disease-related data. Socio-demographic data included gender, age, level of education achieved, area of residence and household income. Behavioural data included smoking status and physical activity [24]. Disease-related data included duration of diabetes, modality of treatment, hypoglycaemia symptoms in the last month, anxiety [25], depression [26], cognitive impairment [27], neuropathy [28] and history of diabetes complications and comorbidities [23].

HRQoL was assessed using the EuroQol 5-Dimension 5-Level (EQ-5D-5L). The EQ-5D-5L is a generic preference-based health status measuring tool [8]. It provides information on health status on five dimensions: mobility, self-care, usual activities, pain-discomfort and anxiety-depression. Each dimension has five possible responses: no problems, slight problems, moderate problems, serious problems and inability or extreme problems. Using a scoring algorithm, the scores in the dimensions can be converted to a single index-based utility score (utility index) [8]. The United Kingdom scoring algorithm is often used when country-specific weights are not available and has been used for the Saudi population previously [20]. The health utility score usually lies between zero and one, where zero indicates very poor health status (death), and one indicates a perfect health status. A score below zero indicates a health status worse than death. The visual analogue scale (VAS) is another part of the EQ-5D-5L instrument in which respondents can use a 20-centimetre VAS to subjectively rate their health status at that day between zero (worst imaginable health status) and 100 (best imaginable health state). The establishers' permissions to use the EQ$5 \mathrm{D}-5 \mathrm{~L}$ and the other tools were obtained.

At the end of the interview, the participants' blood pressure, height and weight were measured in a standardised way [23]. Then, the participants' medical records were reviewed for recent lab test results, currently prescribed medications, and documented diagnosis of hypertension, coronary artery disease, stroke and/or retinopathy.

\section{Operational definitions}

Body mass index (BMI) was categorised according to the current World Health Organization guidelines into normal $\left(<25.0 \mathrm{~kg} / \mathrm{m}^{2}\right)$, pre-obesity $(25.0-$ $\left.29.9 \mathrm{~kg} / \mathrm{m}^{2}\right)$ and obesity $\left(\geq 30.0 \mathrm{~kg} / \mathrm{m}^{2}\right)$. Using the Global Physical Activity Questionnaire [24], the total number of minutes of physical activity per week was categorised into $\geq 150$ minutes and $<150$ minutes [29]. The number of hours spent in sitting position per day were categorized into $<10$ hours and $\geq 10$ hours [30]. Based on haemoglobin A1c (HbA1c) level, glycaemic control was categorised into good control (HbA1c $<7.0 \%)$ and poor control (HbA1c $\geq 7.0 \%)$ [29]. 'Hypoglycaemia symptoms in the last month' was defined as any symptoms of mild or severe hypoglycaemia including feeling hungry, trembling or shakiness, sweating, confusion, difficulty concentrating, and loss of consciousness. 'Hypertension' was defined as either: documented diagnosis of hypertension, taking antihypertension medications, or three previous high blood pressure measures (systolic $\geq 140 \mathrm{mmHg}$ or diastolic $\geq 90 \mathrm{mmHg}$ ) [29]. 'Coronary artery disease' was defined as documented diagnosis of coronary artery disease, taking medication for coronary artery disease, or underwent a procedure for coronary artery disease. 'Stroke' was defined as documented diagnosis of irreversible cerebrovascular accident. 'Diabetic foot' was defined as a history of lower extremity ulcers or amputations. 'Retinopathy' was defined as documented diagnosis of retinopathy, or the participant had been told by an ophthalmologist that he or she had retinopathy. 'Diabetes peripheral neuropathy' was defined as a score of seven or more using the Michigan Neuropathy Screening Instrument [28]. 'Renal impairment' was defined as estimated glomerular filtration rate (eGFR) $\leq 60 \mathrm{ml} / \mathrm{min} / 1.73 \mathrm{~m}^{2}$ which was calculated from serum creatinine using the Chronic Kidney Disease Epidemiology Collaboration (CKD-EPI) equation [31]. 'Depression' was defined as a score of three and more in the Patient Health Questionnaire-2 (PHQ-2) [26]. 'Anxiety' was defined as a score of three and more in the Generalized Anxiety Disorder Scale (GAD-2) [25]. 'Impaired cognitive function' was defined as a score of $\leq 22$ in the Rowland Universal Dementia Assessment Scale (RUDAS) [27].

\section{Data Analysis}

Stata SE version 15.0 was used for data analysis. The United Kingdom scoring algorithm was used to convert the scores in the dimensions to a single indexbased utility score. The original five responses to the EQ-5D-5L dimensions were dichotomized to 'no problems' and 'any problems'. The mean and standard deviation or frequency and percentages were used to summarise data. The univariate association between potential risk factors with the EQ-5D-5L dimensions, utility index and VAS score were assessed using chi-square, t-test or analysis of variance (ANOVA). Based on a literature review and clinical judgment, age, gender, education, household income, location of residence, smoking, physical activity per week, length of sitting time per day, duration of diabetes, number of medications, hypoglycaemia events, insulin use, BMI, HbA1c, hypertension, diabetes complications, anxiety, depression and cognitive impairment were identified as factors with potential association with HRQoL. Of these variables, diabetes duration, insulin use, BMI, HbA1c, neuropathy, kidney disease, and cognitive impairment had missing values which ranged between $0.09 \%$ for diabetes duration and $11.8 \%$ for cognitive impairment. Missing data was imputed five times using the Ice Chained Equation method [32].

To identify factors associated with the EQ-5D-5L dimensions, utility index and the VAS score multiple (logistic or linear) regression with bootstrap resampling with replacement was used [33,34]. From each of the five imputed samples, 5000 samples, each of the same size as the original sample, were randomly drawn with replacement (for a total of 25,000 bootstrap samples). Multiple regression was run for each of the bootstrap samples, and variables that appeared 
as significantly associated with each of the outcomes were recorded. The percentage of times each variable appeared as significant ( $p$-value $\leq 0.05)$ in all the 25,000 samples was calculated. The variables with a percentage of appearance of $50 \%$ or higher were added to a multiple (logistic or linear) regression model, along with age and gender to adjust for possible confounding effects. The adjusted odds ratio or beta coefficient, their $95 \%$ confidence interval, and the $\mathrm{p}$ value for the risk factors associated with the study outcomes were reported.

\section{Results}

\section{General characteristics}

A total of 1,121 persons participated in the study; however, the records of 10 participants $(0.9 \%)$ were substantially incomplete and were excluded ( $n=1,111)$. The mean age was 57.6 ( \pm 11.1 ) years, and $65.2 \%$ (724) of the participants were females. Of the study participants, $51.2 \%(569)$ had achieved primary education or were illiterate, $31.0 \%$ (344) had achieved intermediate or tertiary education and $17.8 \%$ (198) had achieved university education. The mean duration of diabetes was $13.9( \pm 8.4)$ years. The overall mean utility index was $0.739 \pm 0.261$, while the mean VAS score was $69.8 \pm 18.4$. Of the participants, $51.0 \%$ had problems with mobility, $12.3 \%$ had problems with self-care, $30.5 \%$ had problems with usual activity, $51.6 \%$ had problems with pain-discomfort and $45.8 \%$ had problems with anxiety-depression.

\section{Unadjusted association}

Table 1 summarises participants' demographic and behavioural characteristics according to the EQ-5D-5L dimensions, utility index, and VAS score. Secondary level education and lower, physical activity $<150$ minutes per week, and sitting for more than 10 hours per day were associated with lower utility index and VAS score, as well as with higher prevalence of problems with all of the EQ-5D-5L dimensions. The prevalence of problems with mobility, usual activities, and pain-discomfort was higher with advanced age, female gender and lower household income, while advanced age and remote area of residence were associated with problems with self-care. Higher prevalence of problems with anxiety-depression was associated with lower household income and ever having smoked. Advanced age, female gender and low household income were associated with lower mean utility index and VAS score, while remote location of residence was associated with lower utility index but not VAS score. 
Table 1

Demographic and behavioural characteristics by EQ-5D dimensions, utility index, and VAS

\begin{tabular}{|c|c|c|c|c|c|c|c|c|c|c|c|c|c|c|}
\hline \multirow[t]{3}{*}{ Variable } & \multicolumn{10}{|c|}{ EQ-5D Domains } & \multirow{2}{*}{\multicolumn{2}{|c|}{$\begin{array}{l}\text { Utility index } \\
0.739 \pm 0.261\end{array}$}} & \multirow{2}{*}{\multicolumn{2}{|c|}{$\begin{array}{l}\text { VAS } \\
69.8 \pm 18.4\end{array}$}} \\
\hline & \multicolumn{2}{|c|}{$\begin{array}{l}\text { Any problems } \\
\text { with mobility } \\
51.0 \% \text { ( } n= \\
566)\end{array}$} & \multicolumn{2}{|c|}{$\begin{array}{l}\text { Any problems } \\
\text { with self-care } \\
12.3 \%(n= \\
137)\end{array}$} & \multicolumn{2}{|c|}{$\begin{array}{l}\text { Any problems } \\
\text { with usual } \\
\text { activities } \\
30.5 \%(n=339)\end{array}$} & \multicolumn{2}{|c|}{$\begin{array}{l}\text { Any problems } \\
\text { with pain- } \\
\text { discomfort } \\
51.6 \%(n=573)\end{array}$} & \multicolumn{2}{|c|}{$\begin{array}{l}\text { Any problems with } \\
\text { anxiety-depression } \\
45.8 \%(n=509)\end{array}$} & & & & \\
\hline & $\%$ & $\begin{array}{l}\mathrm{P}- \\
\text { value }\end{array}$ & $\%$ & $\begin{array}{l}\mathrm{P} \text { - } \\
\text { value }\end{array}$ & $\%$ & P-value & $\%$ & P-value & $\%$ & P-value & $\begin{array}{l}\text { Mean } \\
\pm S D\end{array}$ & $\begin{array}{l}\text { P- } \\
\text { value }\end{array}$ & $\begin{array}{l}\text { Mean } \\
\pm S D\end{array}$ & $\begin{array}{l}\mathrm{P} \text { - } \\
\text { value }\end{array}$ \\
\hline \multicolumn{15}{|l|}{ Age } \\
\hline$<46$ years & 27.7 & $<.001$ & 8.0 & $<.001$ & 13.1 & $<0.001$ & 27.7 & $<0.001$ & 43.8 & 0.629 & $\begin{array}{l}0.842 \\
\pm \\
0.197\end{array}$ & $\begin{array}{l}< \\
0.001\end{array}$ & $\begin{array}{l}76.1 \\
\pm 16.3\end{array}$ & $\begin{array}{l}<.001 \\
0.00\end{array}$ \\
\hline $46-60$ years & 49.7 & & 9.4 & & 26.8 & & 51.8 & & 47.2 & & $\begin{array}{l}0.745 \\
\pm \\
0.259\end{array}$ & & $\begin{array}{l}69.3 \\
\pm 18.5\end{array}$ & \\
\hline$>60$ years & 39.1 & & 18.2 & & 42.2 & & 59.6 & & 44.5 & & $\begin{array}{l}0.694 \\
\pm \\
0.273\end{array}$ & & $\begin{array}{l}68.2 \\
\pm 18.4\end{array}$ & \\
\hline \multicolumn{15}{|l|}{ Gender: } \\
\hline Female & 54.3 & 0.011 & 11.1 & 0.076 & 34.4 & $<0.001$ & 56.2 & $<0.001$ & 47.7 & 0.093 & $\begin{array}{l}0.709 \\
\pm \\
0.285\end{array}$ & $<.001$ & $\begin{array}{l}66.3 \\
\pm 18.4\end{array}$ & $\begin{array}{l}<.001 \\
0.00\end{array}$ \\
\hline Male & 46.3 & & 14.7 & & 23.3 & & 42.9 & & 42.4 & & $\begin{array}{l}0.795 \\
\pm \\
0.197\end{array}$ & & $\begin{array}{l}76.2 \\
\pm 16.3\end{array}$ & \\
\hline \multicolumn{15}{|l|}{ Education } \\
\hline $\begin{array}{l}\text { University } \\
\text { /college }\end{array}$ & 33.8 & $<.001$ & 6.6 & 0.006 & 16.2 & $<0.001$ & 36.9 & $<0.001$ & 34.3 & $<0.001$ & $\begin{array}{l}0.851 \\
\pm \\
0.115\end{array}$ & $<.001$ & $\begin{array}{l}75.7 \\
\pm 16.2\end{array}$ & $<.001$ \\
\hline $\begin{array}{l}\text { Up to secondary } \\
\text { level }\end{array}$ & 54.7 & & 13.6 & & 33.6 & & 54.8 & & 48.3 & & $\begin{array}{l}0.715 \\
\pm \\
0.273\end{array}$ & & $\begin{array}{l}68.5 \\
\pm 18.6\end{array}$ & \\
\hline \multicolumn{15}{|l|}{$\begin{array}{l}\text { Location of } \\
\text { residence }\end{array}$} \\
\hline Urban & 50.7 & 0.934 & 11.1 & $\begin{array}{l}< \\
0.001\end{array}$ & 29.8 & 0.299 & 50.2 & 0.052 & 44.6 & 0.109 & $\begin{array}{l}0.747 \\
\pm \\
0.252\end{array}$ & 0.003 & $\begin{array}{l}70.2 \\
\pm 18.0\end{array}$ & 0.072 \\
\hline Rural & 52.6 & & 13.7 & & 32.6 & & 56.8 & & 52.6 & & $\begin{array}{l}0.724 \\
\pm \\
0.268\end{array}$ & & $\begin{array}{l}66.0 \\
\pm 19.3\end{array}$ & \\
\hline Remote & 51.6 & & 29.0 & & 38.7 & & 64.5 & & 54.8 & & $\begin{array}{l}0.631 \\
\pm \\
0.355\end{array}$ & & $\begin{array}{l}68.0 \\
\pm 22.1\end{array}$ & \\
\hline \multicolumn{15}{|l|}{$\begin{array}{l}\text { Household } \\
\text { income }\end{array}$} \\
\hline$<6001$ SAR & 54.5 & 0.029 & 14.0 & 0.115 & 36.2 & $<0.001$ & 57.6 & $<0.001$ & 51.8 & $<0.001$ & $\begin{array}{l}0.685 \\
\pm \\
0.303\end{array}$ & $\begin{array}{l}< \\
0.001\end{array}$ & $\begin{array}{l}67.0 \\
\pm 18.6\end{array}$ & $\begin{array}{l}<.001 \\
0.00\end{array}$ \\
\hline$\geq 6001 \mathrm{SAR}$ & 47.9 & & 10.9 & & 25.6 & & 46.4 & & 40.7 & & $\begin{array}{l}0.786 \\
\pm \\
0.208\end{array}$ & & $\begin{array}{l}72.1 \\
\pm 17.9\end{array}$ & \\
\hline \multicolumn{15}{|l|}{ Ever smoked } \\
\hline No & 50.4 & 0.463 & 12.3 & 0.991 & 30.6 & 0.932 & 51.8 & 0.804 & 42.7 & $<0.001$ & $\begin{array}{l}0.743 \\
\pm \\
0.266\end{array}$ & 0.312 & $\begin{array}{l}69.9 \\
\pm 18.8\end{array}$ & 0.469 \\
\hline Yes & 53.3 & & 12.3 & & 30.3 & & 50.8 & & 60.5 & & $\begin{array}{l}0.722 \\
\pm \\
0.234\end{array}$ & & $\begin{array}{l}68.9 \\
\pm 16.5\end{array}$ & \\
\hline
\end{tabular}




\begin{tabular}{|c|c|c|c|c|c|c|c|c|c|c|c|c|c|c|}
\hline \multirow[t]{3}{*}{ Variable } & \multicolumn{10}{|c|}{ EQ-5D Domains } & \multirow{2}{*}{\multicolumn{2}{|c|}{$\begin{array}{l}\text { Utility index } \\
0.739 \pm 0.261\end{array}$}} & \multirow{2}{*}{\multicolumn{2}{|c|}{$\begin{array}{l}\text { VAS } \\
69.8 \pm 18.4\end{array}$}} \\
\hline & \multicolumn{2}{|c|}{$\begin{array}{l}\text { Any problems } \\
\text { with mobility } \\
51.0 \%(\mathrm{n}= \\
566)\end{array}$} & \multicolumn{2}{|c|}{$\begin{array}{l}\text { Any problems } \\
\text { with self-care } \\
12.3 \%(n= \\
137)\end{array}$} & \multicolumn{2}{|c|}{$\begin{array}{l}\text { Any problems } \\
\text { with usual } \\
\text { activities } \\
30.5 \%(n=339)\end{array}$} & \multicolumn{2}{|c|}{$\begin{array}{l}\text { Any problems } \\
\text { with pain- } \\
\text { discomfort } \\
51.6 \%(n=573)\end{array}$} & \multicolumn{2}{|c|}{$\begin{array}{l}\text { Any problems with } \\
\text { anxiety-depression } \\
45.8 \%(n=509)\end{array}$} & & & & \\
\hline & $\%$ & $\begin{array}{l}\text { P- } \\
\text { value }\end{array}$ & $\%$ & $\begin{array}{l}\mathrm{P} \text { - } \\
\text { value }\end{array}$ & $\%$ & P-value & $\%$ & P-value & $\%$ & P-value & $\begin{array}{l}\text { Mean } \\
\pm S D\end{array}$ & $\begin{array}{l}\mathrm{P} \text { - } \\
\text { value }\end{array}$ & $\begin{array}{l}\text { Mean } \\
\pm S D\end{array}$ & $\begin{array}{l}\mathrm{P} \text { - } \\
\text { value }\end{array}$ \\
\hline \multicolumn{15}{|l|}{$\begin{array}{l}\text { Physical } \\
\text { activity }\end{array}$} \\
\hline$\geq 150 \mathrm{~min} /$ week & 27.4 & $<.001$ & 4.0 & $<.001$ & 12.8 & $<0.001$ & 33.8 & $<0.001$ & 35.7 & $<0.001$ & $\begin{array}{l}0.855 \\
\pm \\
0.167\end{array}$ & $<.001$ & $\begin{array}{l}74.0 \\
\pm 16.4\end{array}$ & $<.001$ \\
\hline$<150 \mathrm{~min} /$ week & 60.8 & & 15.8 & & 37.9 & & 59.0 & & 50.1 & & $\begin{array}{l}0.690 \\
\pm \\
0.278\end{array}$ & & $\begin{array}{l}68.0 \\
\pm 18.9\end{array}$ & \\
\hline \multicolumn{15}{|l|}{ Sitting time } \\
\hline $\begin{array}{l}<10 \text { hours per } \\
\text { day }\end{array}$ & 46.0 & $<.001$ & 9.4 & $<.001$ & 25.5 & $<0.001$ & 47.1 & $<0.001$ & 42.8 & $<0.001$ & $\begin{array}{l}0.778 \\
\pm \\
0.220\end{array}$ & $<.001$ & $\begin{array}{l}71.5 \\
\pm 17.5\end{array}$ & $<.001$ \\
\hline $\begin{array}{l}\geq 10 \text { hours per } \\
\text { day }\end{array}$ & 74.5 & & 26.6 & & 54.7 & & 72.9 & & 60.4 & & $\begin{array}{l}0.554 \\
\pm \\
0.351\end{array}$ & & $\begin{array}{l}61.6 \\
\pm 20.3\end{array}$ & \\
\hline
\end{tabular}

Table 2 shows disease-related and clinical characteristics according to the EQ-5D-5L dimensions, utility index, and VAS score. Longer duration of diabetes, insulin use, hypoglycaemia events in the last month, hypertension, diabetic foot, neuropathy, and retinopathy were associated with higher prevalence of problems with all of the EQ-5D-5L dimensions, as well as with lower utility index and VAS score. Coronary artery disease has a similar effect on the EQ-5D-5L dimensions utility index, but not on VAS score. High BMI was associated with lower utility index and VAS score, as well as problems with all of the EQ-5D-5L dimensions except self-care. The prevalence of problems with mobility increased with the number of medications, HbA1c $\geq 7 \%$, stroke, renal disease, depression and cognitive impairment, while problems with self-care were associated with stroke, renal disease and impaired cognitive function. HbA1c $\geq 7 \%$, stroke, renal disease, depression and cognitive impairment were associated with higher prevalence of problems with usual activities. Higher prevalence of problems with pain-discomfort was associated with the number of medications, $\mathrm{HbA} 1 \mathrm{c} \geq 7 \%$, renal disease, depression, and anxiety, while higher prevalence of problems with anxiety-depression was associated with stroke. Other factors associated with lower mean utility index and VAS score were the number of medications, $\mathrm{HbA} 1 \mathrm{c} \geq 7 \%$, renal disease, depression, anxiety and cognitive impairment. Stroke was associated with a lower utility index. 
Disease related and clinical characteristics by EQ-5D dimensions, utility index, and VAS

\begin{tabular}{|c|c|c|c|c|c|c|c|c|c|c|c|c|c|c|}
\hline \multirow[t]{3}{*}{ Variable } & \multicolumn{10}{|c|}{ EQ-5D Domains } & \multirow{2}{*}{\multicolumn{2}{|c|}{$\begin{array}{l}\text { Utility index } \\
0.739 \pm 0.261\end{array}$}} & \multirow{2}{*}{\multicolumn{2}{|c|}{$\begin{array}{l}\text { VAS } \\
69.8 \pm 18.4\end{array}$}} \\
\hline & \multicolumn{2}{|c|}{$\begin{array}{l}\text { Any problems } \\
\text { with mobility } \\
51.0 \%(n= \\
566)\end{array}$} & \multicolumn{2}{|c|}{$\begin{array}{l}\text { Any problems } \\
\text { with self-care } \\
12.3 \%(n= \\
137)\end{array}$} & \multicolumn{2}{|c|}{$\begin{array}{l}\text { Any problems } \\
\text { with usual } \\
\text { activities } \\
30.5 \%(n=339)\end{array}$} & \multicolumn{2}{|c|}{$\begin{array}{l}\text { Any problems } \\
\text { with pain- } \\
\text { discomfort } \\
51.6 \%(n=573)\end{array}$} & \multicolumn{2}{|c|}{$\begin{array}{l}\text { Any problems } \\
\text { with anxiety- } \\
\text { depression } \\
45.8 \%(n=509)\end{array}$} & & & & \\
\hline & $\%$ & $\begin{array}{l}\text { P- } \\
\text { value }\end{array}$ & $\%$ & $\begin{array}{l}\text { P- } \\
\text { value }\end{array}$ & $\%$ & P-value & $\%$ & P-value & $\%$ & P-value & $\begin{array}{l}\text { Mean } \\
\pm S D\end{array}$ & $\begin{array}{l}\mathrm{P} \text { - } \\
\text { value }\end{array}$ & $\begin{array}{l}\text { Mean } \\
\pm S D\end{array}$ & $\begin{array}{l}\mathrm{P} \text { - } \\
\text { value }\end{array}$ \\
\hline \multicolumn{15}{|l|}{ Diabetes duration } \\
\hline$\leq 10$ years & 42.6 & $<.001$ & 8.6 & 0.004 & 22.6 & $<0.001$ & 41.2 & $<0.001$ & 41.0 & 0.011 & $\begin{array}{l}0.789 \\
\pm \\
0.222\end{array}$ & $<.001$ & $\begin{array}{l}71.4 \\
\pm 17.4\end{array}$ & 0.025 \\
\hline$>10$ years & 55.9 & & 14.5 & & 35.2 & & 57.8 & & 48.8 & & $\begin{array}{l}0.709 \\
\pm \\
0.278\end{array}$ & & $\begin{array}{l}68.8 \\
\pm 18.9\end{array}$ & \\
\hline \multicolumn{15}{|l|}{ Insulin use } \\
\hline No & 45.5 & $<.001$ & 7.5 & $<.001$ & 23.7 & $<0.001$ & 44.5 & $<0.001$ & 42.4 & 0.009 & $\begin{array}{l}0.784 \\
\pm \\
0.219\end{array}$ & $<.001$ & $\begin{array}{l}72.7 \\
\pm 17.2\end{array}$ & $\begin{array}{l}<.001 \\
0.00\end{array}$ \\
\hline Yes & 57.7 & & 18.2 & & 38.9 & & 60.3 & & 50.2 & & $\begin{array}{l}0.682 \\
\pm \\
0.296\end{array}$ & & $\begin{array}{l}66.1 \\
\pm 19.1\end{array}$ & \\
\hline \multicolumn{15}{|l|}{$\begin{array}{l}\text { Number of } \\
\text { medications }\end{array}$} \\
\hline $0-2$ medications & 41.4 & 0.014 & 8.1 & 0.247 & 24.7 & 0.134 & 39.1 & 0.001 & 39.1 & 0.154 & $\begin{array}{l}0.800 \\
\pm \\
0.235\end{array}$ & 0.004 & $\begin{array}{l}72.7 \\
\pm 18.0\end{array}$ & 0.027 \\
\hline $3-4$ medications & 51.3 & & 12.7 & & 29.5 & & 52.1 & & 47.7 & & $\begin{array}{l}0.738 \\
\pm \\
0.254\end{array}$ & & $\begin{array}{l}70.2 \\
\pm 17.6\end{array}$ & \\
\hline 5 and more & 54.3 & & 12.4 & & 32.7 & & 54.9 & & 46.3 & & $\begin{array}{l}0.726 \\
\pm \\
0.260\end{array}$ & & $\begin{array}{l}68.5 \\
\pm 18.7\end{array}$ & \\
\hline \multicolumn{15}{|l|}{$\begin{array}{l}\text { Hypoglycaemia } \\
\text { event during last } \\
\text { month }\end{array}$} \\
\hline No & 47.4 & 0.005 & 9.7 & 0.002 & 26.2 & $<0.001$ & 45.3 & $<0.001$ & 39.7 & $<0.001$ & $\begin{array}{l}0.773 \\
\pm \\
0.244\end{array}$ & $<.001$ & $\begin{array}{l}71.3 \\
\pm 17.7\end{array}$ & 0.001 \\
\hline Yes & 55.9 & & 16.0 & & 36.5 & & 60.1 & & 54.2 & & $\begin{array}{l}0.692 \\
\pm \\
0.667\end{array}$ & & $\begin{array}{l}67.6 \\
\pm 19.1\end{array}$ & \\
\hline \multicolumn{15}{|l|}{ Body mass index } \\
\hline Underweight/normal & 38.9 & $<.001$ & 11.5 & 0.106 & 25.7 & $<0.001$ & 42.5 & $<0.001$ & 43.4 & $<0.001$ & $\begin{array}{l}0.796 \\
\pm \\
0.192\end{array}$ & $\dot{0.001}$ & $\begin{array}{l}74.3 \\
\pm 17.0\end{array}$ & $\dot{0.001}$ \\
\hline Pre-obesity & 40.3 & & 9.0 & & 21.3 & & 41.7 & & 36.3 & & $\begin{array}{l}0.812 \\
\pm \\
0.195\end{array}$ & & $\begin{array}{l}73.1 \\
\pm 17.3\end{array}$ & \\
\hline Obesity & 57.4 & & 13.8 & & 35.2 & & 57.4 & & 50.3 & & $\begin{array}{l}0.698 \\
\pm \\
0.286\end{array}$ & & $\begin{array}{l}67.6 \\
\pm 18.4\end{array}$ & \\
\hline \multicolumn{15}{|l|}{ Glycaemic control } \\
\hline $\mathrm{HbA} 1 \mathrm{c}<7 \%$ & 45.3 & 0.029 & 9.9 & 0.161 & 24.7 & 0.023 & 44.5 & 0.008 & 43.7 & 0.402 & $\begin{array}{l}0.773 \\
\pm \\
0.235\end{array}$ & 0.017 & $\begin{array}{l}71.8 \\
\pm 16.5\end{array}$ & 0.029 \\
\hline $\mathrm{HbA} 1 \mathrm{c} \geq 7 \%$ & 53.0 & & 13.2 & & 32.1 & & 53.9 & & 46.7 & & $\begin{array}{l}0.729 \\
\pm \\
0.265\end{array}$ & & $\begin{array}{l}69.0 \\
\pm 18.9\end{array}$ & \\
\hline
\end{tabular}




\begin{tabular}{|c|c|c|c|c|c|c|c|c|c|c|c|c|c|c|}
\hline \multirow[t]{3}{*}{ Variable } & \multicolumn{10}{|c|}{ EQ-5D Domains } & \multirow{2}{*}{\multicolumn{2}{|c|}{$\begin{array}{l}\text { Utility index } \\
0.739 \pm 0.261\end{array}$}} & \multirow{2}{*}{\multicolumn{2}{|c|}{$\begin{array}{l}\text { VAS } \\
69.8 \pm 18.4\end{array}$}} \\
\hline & \multicolumn{2}{|c|}{$\begin{array}{l}\text { Any problems } \\
\text { with mobility } \\
51.0 \% \text { ( } n= \\
566)\end{array}$} & \multicolumn{2}{|c|}{$\begin{array}{l}\text { Any problems } \\
\text { with self-care } \\
12.3 \%(n= \\
137)\end{array}$} & \multicolumn{2}{|c|}{$\begin{array}{l}\text { Any problems } \\
\text { with usual } \\
\text { activities } \\
30.5 \%(n=339)\end{array}$} & \multicolumn{2}{|c|}{$\begin{array}{l}\text { Any problems } \\
\text { with pain- } \\
\text { discomfort } \\
51.6 \%(n=573)\end{array}$} & \multicolumn{2}{|c|}{$\begin{array}{l}\text { Any problems } \\
\text { with anxiety- } \\
\text { depression } \\
45.8 \%(n=509)\end{array}$} & & & & \\
\hline & $\%$ & $\begin{array}{l}\mathrm{P} \text { - } \\
\text { value }\end{array}$ & $\%$ & $\begin{array}{l}\mathrm{P} \text { - } \\
\text { value }\end{array}$ & $\%$ & P-value & $\%$ & P-value & $\%$ & P-value & $\begin{array}{l}\text { Mean } \\
\pm S D\end{array}$ & $\begin{array}{l}\mathrm{P} \text { - } \\
\text { value }\end{array}$ & $\begin{array}{l}\text { Mean } \\
\pm S D\end{array}$ & $\begin{array}{l}\mathrm{P} \text { - } \\
\text { value }\end{array}$ \\
\hline \multicolumn{15}{|c|}{ Hypertension } \\
\hline No & 38.7 & $\begin{array}{l}< \\
0.001\end{array}$ & 6.9 & $<.001$ & 21.9 & $<0.001$ & 42.0 & $<0.001$ & 40.8 & 0.029 & $\begin{array}{l}0.796 \\
\pm \\
0.226\end{array}$ & $\begin{array}{l}< \\
0.001\end{array}$ & $\begin{array}{l}74.1 \\
\pm 17.1\end{array}$ & $\dot{0} .001$ \\
\hline Yes & 56.2 & & 14.7 & & 34.2 & & 55.7 & & 47.9 & & $\begin{array}{l}0.715 \\
\pm \\
0.271\end{array}$ & & $\begin{array}{l}67.9 \\
\pm 18.6\end{array}$ & \\
\hline \multicolumn{15}{|c|}{$\begin{array}{l}\text { Coronary artery } \\
\text { disease }\end{array}$} \\
\hline No & 47.9 & $<.001$ & 10.2 & $<.001$ & 27.0 & $<0.001$ & 48.3 & $<0.001$ & 43.6 & 0.001 & $\begin{array}{l}0.761 \\
\pm \\
0.245\end{array}$ & $<.001$ & $\begin{array}{l}70.2 \\
\pm 17.9\end{array}$ & 0.056 \\
\hline Yes & 65.6 & & 22.8 & & 47.6 & & 67.7 & & 56.6 & & $\begin{array}{l}0.632 \\
\pm \\
0.309\end{array}$ & & $\begin{array}{l}67.4 \\
\pm 20.4\end{array}$ & \\
\hline \multicolumn{15}{|c|}{ Diabetic foot } \\
\hline No & 45.5 & $<.001$ & 9.3 & $<.001$ & 26.9 & $<0.001$ & 46.4 & $<0.001$ & 42.8 & $<0.001$ & $\begin{array}{l}0.776 \\
\pm \\
0.233\end{array}$ & $\begin{array}{l}< \\
0.001\end{array}$ & $\begin{array}{l}71.5 \\
\pm 18.1\end{array}$ & $\dot{0.001}$ \\
\hline Yes & 70.6 & & 32.2 & & 50.4 & & 73.4 & & 61.5 & & $\begin{array}{l}0.552 \\
\pm \\
0.340\end{array}$ & & $\begin{array}{l}63.8 \\
\pm 19.5\end{array}$ & \\
\hline \multicolumn{15}{|l|}{ Stroke } \\
\hline No & 49.5 & $<.001$ & 11.5 & $<.001$ & 29.3 & $<0.001$ & 51.0 & 0.062 & 44.8 & $<0.001$ & $\begin{array}{l}0.747 \\
\pm \\
0.254\end{array}$ & $\begin{array}{l}< \\
0.001\end{array}$ & $\begin{array}{l}69.8 \\
\pm 18.2\end{array}$ & 0.477 \\
\hline Yes & 87.8 & & 35.2 & & 63.4 & & 65.9 & & 73.2 & & $\begin{array}{l}0.532 \\
\pm \\
0.351\end{array}$ & & $\begin{array}{l}67.8 \\
\pm 21.6\end{array}$ & \\
\hline \multicolumn{15}{|c|}{ Retinopathy } \\
\hline No & 45.4 & $<.001$ & 8.4 & $\dot{0} 001$ & 23.9 & $<0.001$ & 43.6 & $<0.001$ & 40.9 & $<0.001$ & $\begin{array}{l}0.785 \\
\pm \\
0.223\end{array}$ & $\dot{0} 001$ & $\begin{array}{l}72.1 \\
\pm 17.0\end{array}$ & $\dot{0.001}$ \\
\hline Yes & 58.4 & & 17.7 & & 39.3 & & 62.2 & & 52.3 & & $\begin{array}{l}0.677 \\
\pm \\
0.293\end{array}$ & & $\begin{array}{l}66.7 \\
\pm 19.7\end{array}$ & \\
\hline \multicolumn{15}{|c|}{ Neuropathy } \\
\hline No & 52.9 & $\begin{array}{l}< \\
0.001\end{array}$ & 8.2 & $\begin{array}{l}< \\
0.001\end{array}$ & 23.5 & $<0.001$ & 43.1 & $<0.001$ & 39.9 & $<0.001$ & $\begin{array}{l}0.796 \\
\pm \\
0.218\end{array}$ & $\begin{array}{l}< \\
0.001\end{array}$ & $\begin{array}{l}72.5 \\
\pm 18.0\end{array}$ & $\begin{array}{l}<.001 \\
0.00\end{array}$ \\
\hline Yes & 73.8 & & 29.7 & & 56.9 & & 78.2 & & 67.8 & & $\begin{array}{l}0.536 \\
\pm \\
0.320\end{array}$ & & $\begin{array}{l}62.1 \\
\pm 18.1\end{array}$ & \\
\hline \multicolumn{15}{|c|}{ Renal disease } \\
\hline No & 47.3 & $<.001$ & 9.2 & $\begin{array}{l}< \\
0.001\end{array}$ & 26.2 & $<0.001$ & 48.4 & $<0.001$ & 45.5 & 0.258 & $\begin{array}{l}0.764 \\
\pm \\
0.229\end{array}$ & $\begin{array}{l}<.001 \\
0.001\end{array}$ & $\begin{array}{l}70.8 \\
\pm 17.9\end{array}$ & $<.001$ \\
\hline Yes & 72.1 & & 28.0 & & 55.9 & & 70.2 & & 50.3 & & $\begin{array}{l}0.593 \\
\pm \\
0.359\end{array}$ & & $\begin{array}{l}63.9 \\
\pm 19.8\end{array}$ & \\
\hline
\end{tabular}




\begin{tabular}{|c|c|c|c|c|c|c|c|c|c|c|c|c|c|c|}
\hline \multirow[t]{3}{*}{ Variable } & \multicolumn{10}{|c|}{ EQ-5D Domains } & \multirow{2}{*}{\multicolumn{2}{|c|}{$\begin{array}{l}\text { Utility index } \\
0.739 \pm 0.261\end{array}$}} & \multirow{2}{*}{\multicolumn{2}{|c|}{$\begin{array}{l}\text { VAS } \\
69.8 \pm 18.4\end{array}$}} \\
\hline & \multicolumn{2}{|c|}{$\begin{array}{l}\text { Any problems } \\
\text { with mobility } \\
51.0 \%(n= \\
566)\end{array}$} & \multicolumn{2}{|c|}{$\begin{array}{l}\text { Any problems } \\
\text { with self-care } \\
12.3 \%(n= \\
137)\end{array}$} & \multicolumn{2}{|c|}{$\begin{array}{l}\text { Any problems } \\
\text { with usual } \\
\text { activities } \\
30.5 \%(n=339)\end{array}$} & \multicolumn{2}{|c|}{$\begin{array}{l}\text { Any problems } \\
\text { with pain- } \\
\text { discomfort } \\
51.6 \%(n=573)\end{array}$} & \multicolumn{2}{|c|}{$\begin{array}{l}\text { Any problems } \\
\text { with anxiety- } \\
\text { depression } \\
45.8 \%(n=509)\end{array}$} & & & & \\
\hline & $\%$ & $\begin{array}{l}\mathrm{P} \text { - } \\
\text { value }\end{array}$ & $\%$ & $\begin{array}{l}\text { P- } \\
\text { value }\end{array}$ & $\%$ & P-value & $\%$ & P-value & $\%$ & P-value & $\begin{array}{l}\text { Mean } \\
\pm S D\end{array}$ & $\begin{array}{l}\mathrm{P} \text { - } \\
\text { value }\end{array}$ & $\begin{array}{l}\text { Mean } \\
\pm S D\end{array}$ & $\begin{array}{l}P \text { - } \\
\text { value }\end{array}$ \\
\hline No & 49.2 & 0.011 & 11.1 & 0.006 & 27.4 & $<0.001$ & 48.3 & $<0.001$ & - & - & $\begin{array}{l}0.772 \\
\pm \\
0.239\end{array}$ & $\begin{array}{l}<.001 \\
0.001\end{array}$ & $\begin{array}{l}72.3 \\
\pm 17.1\end{array}$ & $\begin{array}{l}< \\
0.001\end{array}$ \\
\hline Yes & 59.5 & & 18.4 & & 46.0 & & 68.1 & & & & $\begin{array}{l}0.573 \\
\pm \\
0.301\end{array}$ & & $\begin{array}{l}56.8 \\
\pm 19.2\end{array}$ & \\
\hline \multicolumn{15}{|l|}{ Anxiety } \\
\hline No & 50.5 & 0.518 & 11.6 & 0.080 & 29.5 & 0.076 & 48.5 & $<0.001$ & - & - & $\begin{array}{l}0.765 \\
\pm \\
0.243\end{array}$ & $\begin{array}{l}<.001 \\
0.00\end{array}$ & $\begin{array}{l}71.6 \\
\pm 17.5\end{array}$ & $\begin{array}{l}< \\
0.001\end{array}$ \\
\hline Yes & 53.2 & & 16.4 & & 36.3 & & 68.4 & & & & $\begin{array}{l}0.596 \\
\pm \\
0.307\end{array}$ & & $\begin{array}{l}59.3 \\
\pm 19.5\end{array}$ & \\
\hline \multicolumn{15}{|c|}{$\begin{array}{l}\text { Cognitive } \\
\text { impairment }\end{array}$} \\
\hline No & 46.2 & 0.016 & 8.6 & $\begin{array}{l}<.001 \\
0.00\end{array}$ & 26.4 & $<0.001$ & 48.7 & 0.199 & 44.5 & 0.352 & $\begin{array}{l}0.771 \\
\pm \\
0.231\end{array}$ & $\begin{array}{l}<.001 \\
0 .\end{array}$ & $\begin{array}{l}72.4 \\
\pm 18.3\end{array}$ & $\begin{array}{l}< \\
0.001\end{array}$ \\
\hline Yes & 54.8 & & 22.8 & & 39.3 & & 53.3 & & 47.8 & & $\begin{array}{l}0.679 \\
\pm \\
0.329\end{array}$ & & $\begin{array}{l}66.0 \\
\pm 18.0\end{array}$ & \\
\hline
\end{tabular}

\section{Adjusted association}

Results of bootstrap analysis to identify factors that were significantly associated with EQ-5D-5L dimensions, utility index and VAS score are presented in Table 3. Along with age and gender, factors that appeared significantly associated in at least $50 \%$ of the bootstrap samples were used to develop multiple logistic regression models for each of the EQ-5D-5L dimensions (Table 4), and multiple linear regression models for utility index and VAS score (Table 5). 
Table 3

Percentage of appearance of variables as significantly associated with the EQ-5D dimensions, utility index, and VAS

\begin{tabular}{|c|c|c|c|c|c|c|c|}
\hline \multirow[t]{2}{*}{ Variable } & \multicolumn{5}{|l|}{ EQ-5D Domain } & \multirow{2}{*}{$\begin{array}{l}\text { Utility } \\
\text { index }\end{array}$} & \multirow[t]{2}{*}{ VAS } \\
\hline & $\begin{array}{l}\text { Any problems } \\
\text { with mobility }\end{array}$ & $\begin{array}{l}\text { Any problems } \\
\text { with self-care }\end{array}$ & $\begin{array}{l}\text { Any problems with } \\
\text { usual activities }\end{array}$ & $\begin{array}{l}\text { Any problems with } \\
\text { pain -discomfort }\end{array}$ & $\begin{array}{l}\text { Any problems with } \\
\text { anxiety-depression }\end{array}$ & & \\
\hline Age $\geq 61$ years & $66.0 \% *$ & $14.8 \%$ & $93.4 \% *$ & $86.3 \% *$ & $41.6 \%$ & $36.2 \%$ & $18.3 \%$ \\
\hline Female gender & $10.5 \%$ & $97.4 \% *$ & $14.6 \%$ & $11.8 \%$ & $7.2 \%$ & $3.8 \%$ & $100.0 \%$ * \\
\hline $\begin{array}{l}\text { Education up to } \\
\text { secondary level }\end{array}$ & $78.3 \% *$ & $43.3 \%$ & $46.0 \%$ & $32.0 \%$ & $41.7 \%$ & $89.6 \% *$ & $16.2 \%$ \\
\hline $\begin{array}{l}\text { Remote location of } \\
\text { residence }\end{array}$ & $8.0 \%$ & $91.3 \% *$ & $7.1 \%$ & $20.1 \%$ & $6.3 \%$ & $46.7 \%$ & $19.8 \%$ \\
\hline $\begin{array}{l}\text { Household income< } \\
6001 \text { SAR }\end{array}$ & $36.9 \%$ & $61.8 \% *$ & $9.5 \%$ & $5.8 \%$ & $6.2 \%$ & $6.4 \%$ & $28.9 \%$ \\
\hline Ever smoked & $11.7 \%$ & $9.0 \%$ & $5.9 \%$ & $11.7 \%$ & $77.3 \% *$ & $5.1 \%$ & $11.8 \%$ \\
\hline $\begin{array}{l}\text { Physical activity < } \\
150 \mathrm{~min} / \text { week }\end{array}$ & $100.0 \% *$ & $97.2 \% *$ & $99.5 \% *$ & $96.1 \% *$ & $56.4 \% \star$ & $100.0 \% *$ & $18.6 \%$ \\
\hline $\begin{array}{l}\text { Sitting time } \geq 10 \\
\text { hours per day }\end{array}$ & $96.8 \% *$ & $60.3 \% *$ & $90.6 \% *$ & $86.7 \% *$ & $47.5 \%$ & $100.0 \% *$ & $96.6 \% *$ \\
\hline $\begin{array}{l}\text { Diabetes Duration > } \\
10 \text { years }\end{array}$ & $19.6 \%$ & $8.0 \%$ & $5.9 \%$ & $26.3 \%$ & $31.2 \%$ & $9.7 \%$ & $43.4 \%$ \\
\hline Insulin use & $5.7 \%$ & $52.9 \% *$ & $19.9 \%$ & $6.4 \%$ & $7.0 \%$ & $14.8 \%$ & $91.6 \% *$ \\
\hline $\begin{array}{l}\text { Number of } \\
\text { medications } \geq 4 \\
\text { types }\end{array}$ & $10.4 \%$ & $32.8 \%$ & $17.5 \%$ & $8.3 \%$ & $19.6 \%$ & $10.0 \%$ & $30.6 \%$ \\
\hline $\begin{array}{l}\text { Hypoglycaemia } \\
\text { symptoms last } \\
\text { month }\end{array}$ & $12.1 \%$ & $19.4 \%$ & $16.8 \%$ & $57.1 \% *$ & $59.8 \% *$ & $20.3 \%$ & $5.1 \%$ \\
\hline $\begin{array}{l}\text { Body mass index } \geq \\
30.0 \mathrm{~kg} / \mathrm{m}^{2}\end{array}$ & $96.3 \% *$ & $13.7 \%$ & $59.8 \% *$ & $81.6 \% *$ & $48.5 \%$ & $99.0 \% *$ & $26.7 \%$ \\
\hline $\begin{array}{l}\text { Poor glycaemic } \\
\text { control }(\mathrm{HbA} 1 \mathrm{c} \geq 7 \%)\end{array}$ & $19.8 \%$ & $4.8 \%$ & $25.1 \%$ & $21.6 \%$ & $6.6 \%$ & $8.6 \%$ & $7.6 \%$ \\
\hline Hypertension & $60.5 \% *$ & $46.6 \%$ & $8.7 \%$ & $22.9 \%$ & $24.5 \%$ & $22.7 \%$ & $89.2 \% *$ \\
\hline $\begin{array}{l}\text { Coronary artery } \\
\text { disease }\end{array}$ & $17.7 \%$ & $20.5 \%$ & $50.3 \% *$ & $48.2 \%$ & $29.5 \%$ & $64.2 \% *$ & $11.4 \%$ \\
\hline Diabetic foot & $44.6 \%$ & $80.2 \% *$ & $15.8 \%$ & $40.3 \%$ & $13.5 \%$ & $84.9 \% *$ & $24.8 \%$ \\
\hline Stroke & $86.4 \% *$ & $28.6 \%$ & $69.8 \% *$ & $6.5 \%$ & $41.9 \%$ & $69.0 \% *$ & $19.0 \%$ \\
\hline Retinopathy & $4.9 \%$ & $6.8 \%$ & $9.5 \%$ & $21.9 \%$ & $14.8 \%$ & $13.7 \%$ & $15.2 \%$ \\
\hline Neuropathy & $99.6 \% *$ & $98.3 \% *$ & $99.9 \% *$ & $99.9 \% *$ & $79.0 \% *$ & $100.0 \% *$ & $64.8 \% *$ \\
\hline Renal disease & $86.8 \% *$ & $85.6 \% *$ & $98.5 \% *$ & $58.0 \% *$ & $8.0 \%$ & $98.1 \% *$ & $42.8 \%$ \\
\hline Depression & $23.7 \%$ & $23.2 \%$ & $83.8 \% *$ & $24.1 \%$ & - & $99.2 \% *$ & $100.0 \% *$ \\
\hline Anxiety & $38.4 \%$ & $10.3 \%$ & $45.5 \%$ & $55.9 \% *$ & - & $60.6 \% *$ & $79.1 \% *$ \\
\hline $\begin{array}{l}\text { Impaired cognitive } \\
\text { function }\end{array}$ & $41.1 \%$ & $84.2 \% *$ & $8.1 \%$ & $84.5 \% *$ & $14.6 \%$ & $9.5 \%$ & $69.2 \% *$ \\
\hline
\end{tabular}


Table 4

Adjusted association between various risk factors and each of the EQ-5D dimensions

\begin{tabular}{|c|c|c|c|c|c|c|c|c|c|c|c|c|c|c|}
\hline \multirow[t]{2}{*}{ Variable } & \multicolumn{3}{|c|}{$\begin{array}{l}\text { Any problems with } \\
\text { mobility }\end{array}$} & \multicolumn{3}{|c|}{$\begin{array}{l}\text { Any problems } \\
\text { with self-care }\end{array}$} & \multicolumn{3}{|c|}{$\begin{array}{l}\text { Any problems with } \\
\text { usual activities }\end{array}$} & \multicolumn{3}{|c|}{$\begin{array}{l}\text { Any problems with } \\
\text { pain-discomfort }\end{array}$} & \multicolumn{2}{|c|}{$\begin{array}{l}\text { Any problems } \\
\text { anxiety-depres }\end{array}$} \\
\hline & $\begin{array}{l}\text { Odds } \\
\text { ratio }\end{array}$ & $\begin{array}{l}95 \% \\
\mathrm{Cl}\end{array}$ & $\begin{array}{l}\mathrm{P} \text { - } \\
\text { value }\end{array}$ & $\begin{array}{l}\text { Odds } \\
\text { ratio }\end{array}$ & $\begin{array}{l}95 \% \\
\mathrm{Cl}\end{array}$ & $\begin{array}{l}\mathrm{P} \text { - } \\
\text { value }\end{array}$ & $\begin{array}{l}\text { Odds } \\
\text { ratio }\end{array}$ & $\begin{array}{l}95 \% \\
\mathrm{Cl}\end{array}$ & $\begin{array}{l}\mathrm{P} \text { - } \\
\text { value }\end{array}$ & $\begin{array}{l}\text { Odds } \\
\text { ratio }\end{array}$ & $\begin{array}{l}95 \% \\
\mathrm{Cl}\end{array}$ & $\begin{array}{l}P \text { - } \\
\text { value }\end{array}$ & $\begin{array}{l}\text { Odds } \\
\text { ratio }\end{array}$ & $\begin{array}{l}95 \% \\
\mathrm{Cl}\end{array}$ \\
\hline \multicolumn{15}{|l|}{$\begin{array}{l}\text { Age (ref: } \\
<46 \text { years) }\end{array}$} \\
\hline $46-60$ years & 1.5 & $\begin{array}{l}0.9 \\
2.4\end{array}$ & 0.088 & 0.7 & $\begin{array}{l}0.3 \\
1.6\end{array}$ & 0.399 & 1.7 & $\begin{array}{l}0.9 \\
3.1\end{array}$ & 0.108 & 2.2 & $\begin{array}{l}1.4 \\
3.6\end{array}$ & 0.001 & 1.1 & $\begin{array}{l}0.7 \\
1.6\end{array}$ \\
\hline$>60$ years & 1.6 & $\begin{array}{l}1.0 \\
2.8\end{array}$ & 0.051 & 1.0 & $\begin{array}{l}0.4 \\
2.3\end{array}$ & 0.986 & 2.8 & $\begin{array}{l}1.5 \\
5.4\end{array}$ & 0.001 & 3.2 & $\begin{array}{l}1.9 \\
5.3\end{array}$ & $\begin{array}{l}<.001 \\
0.00\end{array}$ & 0.9 & $\begin{array}{l}0.6 \\
1.4\end{array}$ \\
\hline $\begin{array}{l}\text { Female gender } \\
\text { (ref: Male) }\end{array}$ & 0.8 & $\begin{array}{l}0.6, \\
1.1\end{array}$ & 0.140 & 0.5 & $\begin{array}{l}0.3 \\
0.8\end{array}$ & 0.009 & 1.5 & $\begin{array}{l}1.1 \\
2.2\end{array}$ & 0.024 & 1.2 & $\begin{array}{l}0.9 \\
1.7\end{array}$ & 0.172 & 1.1 & $\begin{array}{l}0.8 \\
1.4\end{array}$ \\
\hline $\begin{array}{l}\text { Education up to } \\
\text { secondary level } \\
\text { (ref: university) }\end{array}$ & 1.6 & $\begin{array}{l}1.1 \\
2.4\end{array}$ & 0.023 & - & - & - & - & - & - & - & - & - & - & - \\
\hline $\begin{array}{l}\text { Household } \\
\text { income< } 6001 \\
\text { SAR (ref: } \\
\geq 6001 \text { ) }\end{array}$ & - & - & - & 0.7 & $\begin{array}{l}0.4 \\
1.2\end{array}$ & 0.224 & - & - & - & - & - & - & - & - \\
\hline $\begin{array}{l}\text { Remote area of } \\
\text { residence (ref: } \\
\text { urban / rural) }\end{array}$ & - & - & - & 3.2 & $\begin{array}{l}1.5 \\
7.1\end{array}$ & 0.003 & - & - & - & - & - & - & - & - \\
\hline $\begin{array}{l}\text { Ever smoked } \\
\text { (ref: no) }\end{array}$ & - & - & - & - & - & - & - & - & - & - & - & - & 2.1 & $\begin{array}{l}1.5 \\
2.9\end{array}$ \\
\hline $\begin{array}{l}\text { Physical activity } \\
\text { < } 150 \mathrm{~min} / \text { week } \\
\text { (Ref: } \\
\geq 150 \mathrm{~min} / \text { week) }\end{array}$ & 3.0 & $\begin{array}{l}2.1 \\
4.1\end{array}$ & $<.001$ & 3.2 & $\begin{array}{l}1.6 \\
6.3\end{array}$ & 0.001 & 2.7 & $\begin{array}{l}1.8, \\
4.1\end{array}$ & $\begin{array}{l}< \\
0.001\end{array}$ & 1.8 & $\begin{array}{l}1.3 \\
2.5\end{array}$ & $<.001$ & 1.6 & $\begin{array}{l}1.2 \\
2.2\end{array}$ \\
\hline $\begin{array}{l}\text { Sitting time } \geq 10 \\
\text { hours per day } \\
\text { (ref: }<10 \\
\text { hours/day) }\end{array}$ & 2.0 & $\begin{array}{l}1.3 \\
3.2\end{array}$ & 0.002 & 1.8 & $\begin{array}{l}1.0 \\
3.0\end{array}$ & 0.039 & 2.2 & $\begin{array}{l}1.4 \\
3.4\end{array}$ & $<0.001$ & 2.0 & $\begin{array}{l}1.3 \\
3.1\end{array}$ & 0.003 & - & - \\
\hline Using insulin & - & - & - & 1.4 & $\begin{array}{l}0.9 \\
2.3\end{array}$ & 0.132 & - & - & - & - & - & - & - & - \\
\hline $\begin{array}{l}\text { Hypoglycaemia } \\
\text { events last } \\
\text { month (ref: no) }\end{array}$ & - & - & - & - & - & - & - & - & - & 1.3 & $\begin{array}{l}1.0 \\
1.8\end{array}$ & 0.062 & 1.3 & $\begin{array}{l}1.0 \\
1.7\end{array}$ \\
\hline \multicolumn{15}{|l|}{$\begin{array}{l}\text { BMI }(\mathrm{ref}< \\
\left.25.0 \mathrm{~kg} / \mathrm{m}^{2}\right)\end{array}$} \\
\hline $25.0-29.9 \mathrm{~kg} / \mathrm{m}^{2}$ & 0.9 & $\begin{array}{l}0.5 \\
1.4\end{array}$ & 0.580 & - & - & - & 0.7 & $\begin{array}{l}0.4 \\
1.3\end{array}$ & 0.272 & 1.0 & $\begin{array}{l}0.6 \\
1.7\end{array}$ & 0.961 & - & - \\
\hline$\geq 30.0 \mathrm{~kg} / \mathrm{m}^{2}$ & 1.5 & $\begin{array}{l}0.9 \\
2.4\end{array}$ & 0.113 & - & - & - & 1.1 & $\begin{array}{l}0.6 \\
1.9\end{array}$ & 0.708 & 1.6 & $\begin{array}{l}1.0 \\
2.6\end{array}$ & 0.048 & - & - \\
\hline $\begin{array}{l}\text { Hypertension } \\
\text { (ref: no) }\end{array}$ & 1.5 & $\begin{array}{l}1.1 \\
2.0\end{array}$ & 0.022 & - & - & - & - & - & - & - & - & - & - & - \\
\hline $\begin{array}{l}\text { Coronary artery } \\
\text { disease (ref: no) }\end{array}$ & - & - & - & - & - & - & 1.7 & $\begin{array}{l}1.1 \\
2.5\end{array}$ & 0.015 & - & - & - & - & - \\
\hline $\begin{array}{l}\text { Diabetic foot } \\
\text { (ref: no) }\end{array}$ & - & - & - & 2.2 & $\begin{array}{l}1.3 \\
3.8\end{array}$ & 0.004 & - & - & - & - & - & - & - & - \\
\hline Stroke (ref: no) & 4.4 & $\begin{array}{l}1.5 \\
13.3\end{array}$ & 0.008 & - & - & - & 2.7 & $\begin{array}{l}1.2 \\
6.2\end{array}$ & 0.017 & - & - & - & - & - \\
\hline $\begin{array}{l}\text { Neuropathy (ref: } \\
\text { no) }\end{array}$ & 2.8 & $\begin{array}{l}1.9 \\
4.2\end{array}$ & $\dot{0} 001$ & 3.4 & $\begin{array}{l}2.0 \\
5.6\end{array}$ & $\dot{0} 001$ & 3.2 & $\begin{array}{l}2.2 \\
4.6\end{array}$ & $<.001$ & 3.3 & $\begin{array}{l}2.2 \\
5.0\end{array}$ & $<0.001$ & 2.7 & $\begin{array}{l}1.9 \\
3.8\end{array}$ \\
\hline $\begin{array}{l}\text { Renal } \\
\text { impairment (ref: } \\
\text { no) }\end{array}$ & 2.1 & $\begin{array}{l}1.4 \\
3.3\end{array}$ & 0.001 & 2.4 & $\begin{array}{l}1.4 \\
3.9\end{array}$ & 0.001 & 2.8 & $\begin{array}{l}1.8 \\
4.3\end{array}$ & $<.001$ & 1.8 & $\begin{array}{l}1.2 \\
2.8\end{array}$ & 0.008 & - & - \\
\hline
\end{tabular}




\begin{tabular}{|c|c|c|c|c|c|c|c|c|c|c|c|c|c|c|}
\hline \multirow[t]{2}{*}{ Variable } & \multicolumn{3}{|c|}{$\begin{array}{l}\text { Any problems with } \\
\text { mobility }\end{array}$} & \multicolumn{3}{|c|}{$\begin{array}{l}\text { Any problems } \\
\text { with self-care }\end{array}$} & \multicolumn{3}{|c|}{$\begin{array}{l}\text { Any problems with } \\
\text { usual activities }\end{array}$} & \multicolumn{3}{|c|}{$\begin{array}{l}\text { Any problems with } \\
\text { pain-discomfort }\end{array}$} & \multicolumn{2}{|c|}{$\begin{array}{l}\text { Any problems } \\
\text { anxiety-depres }\end{array}$} \\
\hline & $\begin{array}{l}\text { Odds } \\
\text { ratio }\end{array}$ & $\begin{array}{l}95 \% \\
\mathrm{Cl}\end{array}$ & $\begin{array}{l}\mathrm{P} \text { - } \\
\text { value }\end{array}$ & $\begin{array}{l}\text { Odds } \\
\text { ratio }\end{array}$ & $\begin{array}{l}95 \% \\
\mathrm{Cl}\end{array}$ & $\begin{array}{l}\mathrm{P} \text { - } \\
\text { value }\end{array}$ & $\begin{array}{l}\text { Odds } \\
\text { ratio }\end{array}$ & $\begin{array}{l}95 \% \\
\mathrm{Cl}\end{array}$ & $\begin{array}{l}\mathrm{P}- \\
\text { value }\end{array}$ & $\begin{array}{l}\text { Odds } \\
\text { ratio }\end{array}$ & $\begin{array}{l}95 \% \\
\mathrm{Cl}\end{array}$ & $\begin{array}{l}\mathrm{P} \text { - } \\
\text { value }\end{array}$ & $\begin{array}{l}\text { Odds } \\
\text { ratio }\end{array}$ & $\begin{array}{l}95 \% \\
\mathrm{Cl}\end{array}$ \\
\hline $\begin{array}{l}\text { Depression (ref: } \\
\text { no) }\end{array}$ & - & - & - & - & - & - & 1.6 & $\begin{array}{l}1.0 \\
2.4\end{array}$ & 0.033 & - & - & - & - & - \\
\hline Anxiety (ref: no) & - & - & - & - & - & - & - & - & - & 1.9 & $\begin{array}{l}1.3 \\
3.0\end{array}$ & 0.002 & - & - \\
\hline $\begin{array}{l}\text { Impaired } \\
\text { cognitive } \\
\text { function (ref: no) }\end{array}$ & - & - & - & 2.3 & $\begin{array}{l}1.4, \\
3.7\end{array}$ & 0.001 & - & - & - & 0.7 & $\begin{array}{l}0.5 \\
1.0\end{array}$ & 0.046 & - & - \\
\hline
\end{tabular}

Note: The area under the receiver operational characteristics curve for the models predicting problems with mobility, self-care, usual activities, pain-discomfor anxiety-depression were respectively $75.6 \%, 81.7 \%, 79.1 \%, 74.5 \%, 66.5 \%$.

Table 5

Adjusted association between various risk factors and utility index and VAS

\begin{tabular}{|c|c|c|c|c|c|c|}
\hline \multirow[t]{2}{*}{ Variable } & \multicolumn{3}{|l|}{ Utility index } & \multicolumn{3}{|l|}{ VAS } \\
\hline & Beta coefficient & $95 \% \mathrm{Cl}$ & P-value & Beta coefficient & $95 \% \mathrm{Cl}$ & P-value \\
\hline \multicolumn{7}{|l|}{ Age (ref: <46 years) } \\
\hline $46-60$ years & -0.012 & $-0.054,0.030$ & 0.566 & -2.236 & $-5.471,0.999$ & 0.175 \\
\hline$>60$ years & -0.031 & $-0.077,0.015$ & 0.185 & -2.688 & $-6.242,0.867$ & 0.138 \\
\hline Female gender (ref: Male) & -0.021 & $-0.050,0.008$ & 0.163 & -6.176 & $-8.335,-4.016$ & $<0.001$ \\
\hline Education up to secondary level (ref: university) & -0.056 & $0.092,-0.020$ & 0.003 & - & - & - \\
\hline Physical activity < $150 \mathrm{~min} /$ week (Ref: $\geq 150 \mathrm{~min} /$ week) & -0.074 & $-0.104,-0.043$ & $<0.001$ & - & - & - \\
\hline Sitting time $\geq 10$ hours per day (ref: $<10$ hours/day) & -0.133 & $-0.173,-0.094$ & $<0.001$ & -4.875 & $-7.941,-1.808$ & 0.002 \\
\hline Using insulin & - & - & - & -3.589 & $-5.715,-1.462$ & 0.001 \\
\hline \multicolumn{7}{|l|}{ BMI $\left(r e f<25.0 \mathrm{~kg} / \mathrm{m}^{2}\right)$} \\
\hline $25.0-29.9 \mathrm{~kg} / \mathrm{m}^{2}$ & 0.015 & $-0.032,0.063$ & 0.520 & - & - & - \\
\hline$\geq 30.0 \mathrm{~kg} / \mathrm{m}^{2}$ & -0.043 & $-0.087,0.001$ & 0.056 & - & - & - \\
\hline Hypertension (ref: no) & - & - & - & -4.279 & $-6.607,-1.952$ & $<0.001$ \\
\hline Coronary artery disease (ref: no) & -0.068 & $-0.104,-0.032$ & $<0.001$ & - & - & - \\
\hline Neuropathy (ref: no) & -0.138 & $-0.175,-0.101$ & $<0.001$ & -4.788 & $-7.520,-2.056$ & 0.001 \\
\hline Renal impairment (ref: no) & -0.101 & $-0.140,-0.063$ & $<0.001$ & - & - & - \\
\hline Stroke (ref: no) & -0.146 & $-0.220,-0.071$ & $<0.001$ & - & - & - \\
\hline Diabetic foot (ref: no) & -0.074 & $-0.115,-0.032$ & 0.001 & - & - & - \\
\hline Depression (ref: no) & -0.095 & $-0.136,-0.053$ & $<0.001$ & -10.910 & $-14.187,-8.634$ & $<0.001$ \\
\hline Anxiety (ref: no) & -0.062 & $-0.104,-0.019$ & 0.004 & -4.077 & $-7.437,-0.716$ & 0.017 \\
\hline Impaired cognitive function (ref: no) & - & - & - & -3.226 & $-5.641,-0.810$ & 0.009 \\
\hline
\end{tabular}

The odds of problems with mobility increased by $60 \%$ among people with secondary level education or lower. Long sitting time and physical inactivity increased the odds 2-fold and 3-fold, respectively (Table 4), while hypertension increased the odds by 50\%. Stroke, neuropathy, and renal impairment were associated with 4.4-fold, 2.8-fold, and 2.1-fold increases in the odds of problems with mobility, respectively.

Regarding problems with self-care, the odds decreased by $50 \%$ for female gender, while remote area of residence was associated with a $3.2-$ fold increase in odds. Long sitting time and physical inactivity led to 1.8-fold and 3.2-fold increase in odds, respectively. Diabetic foot, neuropathy, and renal impairment were associated with 2.2-fold, 3.4-fold and 2.4-fold increase in odds, respectively. Impaired cognitive function increased the odds 2.3 -fold.

The odds of problems with usual activity increased by a 2.8 -fold for age over 60 years and by $50 \%$ for being female. Long sitting time and physical inactivity were associated with 2.2-fold and 2.7-fold increase in odds, respectively. The odds of problems with usual activity also increased 1.7-fold for coronary artery disease, 2.7-fold for stroke, 3.2-fold for neuropathy, 2.8-fold for renal impairment and 1.6-fold for depression.

Page 12/16 
With regard to problems with pain-discomfort, the odds increased 2.2-fold for age between 46 and 60 years, and 3.2-fold for age over 60 years. Physical inactivity and long sitting time increased the odds 1.8 -fold and 2.0 -fold respectively, while BMI $\geq 30.0 \mathrm{~kg} / \mathrm{m}^{2}$ increased the odds by $60 \%$. Neuropathy and renal impairment were associated with 3.3-fold and 1.8-fold higher odds, respectively. There was also an increase in the odds by $90 \%$ for anxiety, while impaired cognitive function was associated with $30 \%$ lower odds.

The odds of problems with anxiety-depression was higher by 2.1-fold for those who were current or past smokers and by 1.6 -fold for being physically inactive. Hypoglycaemia events in last month was associated with a 1.3-fold increase in odds, while neuropathy increased the odds of problems with anxietydepression 2.7-fold.

Results of the multiple linear regression analysis for utility index and VAS score are presented in Table 5. Factors significantly associated with lower utility index were low education level, physical inactivity, long sitting time, coronary artery disease, diabetic foot, stroke, neuropathy, renal impairment, depression and anxiety. The model has a coefficient of determination $\left(R^{2}\right)$ of $37.5 \%$. Lower VAS score was associated with being female, long sitting time, use of insulin, hypertension, neuropathy, depression, anxiety and cognitive impairment. The model's $\mathrm{R}^{2}$ was $21.4 \%$.

\section{Discussion}

HRQoL is a vital tool in the assessment of the effect of chronic diseases on quality of life. Diabetes and its related complications can have a substantial effect on HRQoL. The aim of this study was to assess the HRQoL and its associated factors among people with T2DM in Saudi Arabia. Results showed that the HRQoL for people with T2DM in Saudi Arabia was low, especially among females and those who had diabetes complications. A number of lifestyle and other modifiable factors were also found to be associated with low HRQoL.

The mean utility index and VAS score in this study were comparable to a previous single-centre small sample study from Saudi Arabia, which reported a mean utility index of $0.71 \pm 0.22$ and a VAS score of $68.5 \pm 16.8$ [20]. A study from Iran also reported a similar mean utility index; however, the VAS score in that study was lower than that in the present study [35]. Studies from Norway, Japan and Korea reported higher utility indexes ranging from 0.83 to 0.94 [36-38]. Differences in the populations and the healthcare systems may explain the variation in the utility index between these studies and the current study. The highest prevalence of any problems among this study's participants was with pain-discomfort ( $51.6 \%)$, followed by mobility (51.0\%) and then anxietydepression (45.8\%). This is in concordance with the results of studies from Iran, Japan and Norway [35-37].

Advanced age appeared to be associated with problems with usual activities and pain-discomfort but not with utility index. Similar findings regarding the effect of age on utility index were reported in previous studies from Saudi Arabia and Canada [11, 20,39]. In contrast, other studies have shown that advanced age was associated with lower utility index $[6,10,40]$. With advanced age, there may be a reduction in body strength and physical function that affects quality of life.

Female participants in this study had a lower mean utility index compared to male participants. Many previous studies reported similar findings [10, 11, 20, 22, $35,41,42]$, and linked this association to higher rates of physical inactivity and obesity among women $[20,35,42]$. Among our participants, higher proportions of females were physically inactive and were spending more than 10 hours per day in a sitting position compared to males. The prevalence of obesity among females was also higher than that among males. This study also showed that physical inactivity was associated with problems with all of the EQ-5D-5L dimensions, while longer sitting time appeared to have a similar association except for anxiety-depression. Moreover, both physical inactivity and longer sitting time were independently associated with a lower utility index. Our finding about the association between physical inactivity and lower HRQoL is supported by previous studies $[11,15,43,44]$. However, the current study was the first to investigate the association between longer sitting time and lower HRQoL among people with T2DM. Longer sitting time was found to be associated with higher risk of diabetes complications [19], which were strongly linked to lower HRQoL $[15,22,39,40]$. Thus, maintaining HRQoL through increasing physical activity and reducing sitting time should be emphasised in the management of diabetes in Saudi Arabia.

Obesity can affect HRQoL, mainly through its impact on physical function and mental health. There are, however, conflicting reports regarding the association between obesity and HRQoL. This study showed that obesity was associated with higher risk of problems with pain-discomfort; yet, obesity did not appear to be associated with lower utility index. Similar findings were reported in another study [36]. In contrast, some studies have reported a significant association [6, 11]. In the current study, obesity was more prevalent among young compared to old people. Young people were also less likely to have diabetes complications, which may explain why obesity was not associated with a lower utility index. Nevertheless, obesity have been linked to higher risk of diabetes complications among people with T2DM in Saudi Arabia [19]. Therefore, controlling the body weight is an important step to lower the risk of diabetes complications and maintain HRQoL.

Similar to other studies [10,35], the current study showed that a lower level of education was related to problems with mobility and lower HRQoL. Having a higher level of education increases the likelihood of adherence to disease management plans and healthier lifestyle behaviours which lowers the risk of diabetes complications and helps in maintaining HRQoL [45]. Previous studies also reported an association between poor HRQoL and both lower income and rural or remote location of residence $[10,46]$. The results of this study showed that, while remote area of residence was associated with problems with selfcare, household income and the area of residence were not associated with a lower utility index. A similar finding regarding income was reported by another study from Saudi Arabia [11].

This study showed that the duration of diabetes did not affect HRQoL. Previous studies from Saudi Arabia and Iran reported similar findings [11, 20, 35]. The modality of treatment and the number of medications were also not associated with the utility index in this study; however, insulin use was associated with a lower VAS score. The results of previous studies regarding the effect of modality of treatment and the number of medications were conflicting, some studies finding an association [16, 40], and others not [20,35]. 
Fear of hypoglycaemia may not affect HRQoL directly, but it may have an impact on the general quality of life through its negative effect on independence, spontaneity and enjoyment of leisure activities [36]. This study also showed that hypoglycaemia was associated with a higher risk of anxiety-depression and that anxiety and depression were independently related to lower utility index and VAS score. Previous studies also reported an association between HRQoL and both anxiety and depression $[47,48]$. Having smoked also appeared to be associated with problems with anxiety-depression, which may be explained by its known relationship with increased risk of diabetes related complications. People with diabetes are at an increased risk of developing anxiety and depression, and these mental illnesses can negatively affect the control of the disease and increase the risk of diabetes complications [49]. To optimise HRQoL among people with T2DM in Saudi Arabia, screening and management of anxiety and depression should be prioritised among this high-risk population.

Diabetes complications have a strong impact on HRQoL. In the current study, diabetes complications were associated with a 0.16- to 0.31 -point reduction in the mean utility index compared to people without complications (utility index $0.84 \pm 0.17$ ). Further analysis showed that people with macrovascular complications only, and those with microvascular complications only had a mean utility index of $0.76 \pm 0.17$ and $0.73 \pm 0.25$, respectively. For people who had both types of complications, the mean dropped to $0.57 \pm 0.33$. A similar decrement in the mean utility index for those with diabetes complications was reported by other studies $[39,50]$. After adjustment for confounders, diabetes complications remained strongly associated with lower utility index. This is in concordance with the literature, which showed that diabetes complications have a substantial effect on HRQoL $[6,11,15,22,39,40]$. Thus, the prevention of diabetes complications is a key measure in improving the quality of life for people with diabetes.

The results of the current study also showed that diabetes complications affected several dimensions of HRQoL. Neuropathy in particular had an impact on all of the HRQoL dimensions and was also associated with a lower VAS score. A strong association between neuropathy and HRQoL has been reported previously $[5,10]$. Altogether, this indicates that neuropathy is a concerning condition that should be prevented or controlled through adequate screening, treatment and patient education.

Major strengths of this study were the relatively large sample size drawn from three different regions and the examinations of the effect of various demographic, behavioural, and clinical factors on HRQoL using a robust analysis method. The use of the EQ-5D-5L tool to assess HRQoL also adds strength to this study in making it easier to compare its results with those of other national and international studies. The results of this study, however, need to be interpreted in the light of being a cross-sectional, meaning that only association and not causation can be inferred from its results. Nevertheless, this study has identified factors that have strong association with lower HRQoL among people with T2DM in Saudi Arabia. Future research should focus on exploring the identified associations using prospective studies. Healthcare providers and health policy makers should also use this study's findings to develop patient-level interventions and public health strategies to improve the quality of life of people with diabetes in Saudi Arabia.

\section{Conclusions}

To summarise, people with T2DM in Saudi Arabia have low HRQoL, especially among females and those with diabetes complications. In order to improve the quality of life for this high-risk group, an active lifestyle should be encouraged, and anxiety, depression and diabetes complications should be prevented or controlled by patient-level interventions and public health strategies.

\section{Declarations}

\section{Ethics approval and consent to participate}

Ethical approval was obtained from the Monash University Human Research Ethics Committee in Australia and the Research Ethics Committee of the Ministry of Health in Saudi Arabia. All study procedures were carried out in accordance with the principles of the Declaration of Helsinki as revised in 2013. Written informed consent was obtained from all participants.

\section{Consent for publication}

Not applicable.

\section{Availability of data and materials}

The datasets generated and analysed during the current study are available from the corresponding author on reasonable request.

\section{Competing interests}

The authors declare that they have no competing interests.

\section{Funding}

This research did not receive any specific grant from funding agencies in the public, commercial, or not-for-profit sectors. 


\section{Author contributions}

All authors were involved in the conception and design of the study. MJA, NAA, SMA, AMA, GMA, NAB and FAA contributed to the acquisition of data. MJA, AA, and $B B$ contributed to data analysis. MJA, AA, BS, and BB contributed to interpretation of results. MJA and BB drafted the manuscript. All authors critically reviewed the manuscript and approved the final version.

\section{Acknowledgements}

We would like to acknowledge the contribution of Waad Faozi Mahfoud, Hesham J. Alramadhan, Ameera J. Alramadan, Ahmed J. Alramadhan, Talal A. Alzahrani, Hawra H. Alramadhan, Ayat S. Alabdullah, Husam M. Almaramhi, Nawaf F. Almukaibil, Nouf A. Alzaid, Wed A. Alkharras, and Ahmad Alhammad in the data collection for this study.

\section{References}

1. Cho N, Shaw J, Karuranga S, Huang Y, da Rocha Fernandes J, Ohlrogge A, et al. IDF Diabetes Atlas: Global estimates of diabetes prevalence for 2017 and projections for 2045. Diabetes research and clinical practice. 2018;138:271-81.

2. Cho NH, Kirigia J, Mbanya JC, Ogurstova K, Guariguata L, Rathmann W, et al. IDF DIABETES ATLAS Eighth edition 2017. International Diabetes Federation. 2017:1 - 147.

3. Trikkalinou A, Papazafiropoulou AK, Melidonis A. Type 2 diabetes and quality of life. World journal of diabetes. 2017;8(4):120.

4. Control CfD, Prevention, Control CfD, Prevention. Measuring healthy days: Population assessment of health-related quality of life. Atlanta: CDC. 2000:4-6.

5. Janssen M, Lubetkin E, Sekhobo J, Pickard A. The use of the EQ-5D preference-based health status measure in adults with type 2 diabetes mellitus. Diabetic Medicine. 2011;28(4):395-413.

6. Morgan CL, McEwan P, Morrissey M, Peters J, Poole C, Currie C. Characterization and comparison of health-related utility in people with diabetes with various single and multiple vascular complications. Diabetic Medicine. 2006;23(10):1100-5.

7. Speight J, Reaney M, Barnard K. Not all roads lead to Rome-a review of quality of life measurement in adults with diabetes. Diabetic Medicine. 2009;26(4):315-27.

8. Oppe M, Devlin NJ, Szende A. EQ-5D value sets: inventory, comparative review and user guide: Springer; 2007.

9. Grandy S, Fox KM. EQ-5D visual analog scale and utility index values in individuals with diabetes and at risk for diabetes: Findings from the Study to Help Improve Early evaluation and management of risk factors Leading to Diabetes (SHIELD). Health and quality of life outcomes. 2008;6(1):18.

10. Safita N, Islam SMS, Chow CK, Niessen L, Lechner A, Holle R, et al. The impact of type 2 diabetes on health related quality of life in Bangladesh: results from a matched study comparing treated cases with non-diabetic controls. Health and quality of life outcomes. 2016;14(1):129.

11. Al-Shehri AH, Taha AZ, Bahnassy AA, Salah M. Health-related quality of life in type 2 diabetic patients. Annals of Saudi medicine. 2008;28(5):352-60.

12. Akinci F, Yildirim A, Gözü H, Sargın H, Orbay E, Sargın M. Assessment of health-related quality of life (HRQoL) of patients with type 2 diabetes in Turkey. Diabetes research and clinical practice. 2008;79(1):117-23.

13. Maddigan SL, Majumdar SR, Toth EL, Feeny DH, Johnson JA. Health-related quality of life deficits associated with varying degrees of disease severity in type 2 diabetes. Health and quality of life outcomes. 2003;1(1):78.

14. Redekop WK, Koopmanschap MA, Stolk RP, Rutten GE, Wolffenbuttel BH, Niessen LW. Health-related quality of life and treatment satisfaction in Dutch patients with type 2 diabetes. Diabetes care. 2002;25(3):458-63.

15. Jing $X$, Chen J, Dong Y, Han D, Zhao H, Wang X, et al. Related factors of quality of life of type 2 diabetes patients: a systematic review and meta-analysis. Health and quality of life outcomes. 2018;16(1):189.

16. Bradley $C$, Eschwège $E$, de Pablos-Velasco P, Parhofer KG, Simon D, Vandenberghe $H$, et al. Predictors of quality of life and other patient-reported outcomes in the PANORAMA multinational study of people with type 2 diabetes. Diabetes Care. 2018;41(2):267-76.

17. Feng X, Astell-Burt T. Impact of a type 2 diabetes diagnosis on mental health, quality of life, and social contacts: a longitudinal study. BMJ Open Diabetes Research and Care. 2017;5(1):e000198.

18. Alwakeel J, Sulimani R, Al-Asaad H, Al-Harbi A, Tarif N, Al-Suwaida A, et al. Diabetes complications in 1952 type 2 diabetes mellitus patients managed in a single institution. Annals of Saudi medicine. 2008;28(4):260.

19. Alramadan MJ, Magliano DJ, Alhamrani HA, Alramadan AJ, Alameer SM, Amin GM, et al. Lifestyle factors and macro-and micro-vascular complications among people with type 2 diabetes in Saudi Arabia. Diabetes \& Metabolic Syndrome: Clinical Research \& Reviews. 2018;13(1):484-91.

20. AL-Aboudi IS, Hassali MA, Shafie AA, Hassan A, Alrasheedy AA. A cross-sectional assessment of health-related quality of life among type 2 diabetes patients in Riyadh, Saudi Arabia. SAGE open medicine. 2015;3:2050312115610129.

21. Al-Tuwijri AA, Al-Doghether MH, Akturk Z, Al-Megbil TI. Quality of life of people with diabetes attending primary care health centres in Riyadh: bad controlgood quality? Quality in Primary Care. 2007;15(5).

22. Al Hayek AA, Robert AA, Al Saeed A, Alzaid AA, Al Sabaan FS. Factors associated with health-related quality of life among Saudi patients with type 2 diabetes mellitus: a cross-sectional survey. Diabetes \& metabolism journal. 2014;38(3):220-9.

23. Alramadan MJ, Afroz A, Batais MA, Almigbal TH, Alhamrani HA, Albaloshi A, et al. A Study Protocol to Assess the Determinants of Glycaemic Control, Complications and Health Related Quality of Life for People with Type 2 Diabetes in Saudi Arabia. Journal of Health Education Research \& Development. 
2017;5(2):1-6.

24. Armstrong T, Bull F. Development of the world health organization global physical activity questionnaire (GPAQ). Journal of Public Health. 2006;14(2):6670.

25. Skapinakis P. The 2-item Generalized Anxiety Disorder scale had high sensitivity and specificity for detecting GAD in primary care. Evidence Based Medicine. 2007;12(5):149-.

26. Kroenke K, Spitzer RL, Williams JB. The Patient Health Questionnaire-2: validity of a two-item depression screener. Medical care. 2003;41(11):1284-92.

27. Storey JE, Rowland JT, Conforti DA, Dickson HG. The Rowland universal dementia assessment scale (RUDAS): a multicultural cognitive assessment scale. International Psychogeriatrics. 2004;16(01):13-31.

28. Moghtaderi A, Bakhshipour A, Rashidi H. Validation of Michigan neuropathy screening instrument for diabetic peripheral neuropathy. Clinical neurology and neurosurgery. 2006;108(5):477-81.

29. American Diabetes Association. American Diabetes Association Standards of Medical Care in Diabetes-2017. The Journal of Clinical and Applied Research and Education. 2017;40.

30. Parrinello CM, Matsushita K, Woodward M, Wagenknecht LE, Coresh J, Selvin E. Risk prediction of major complications in individuals with diabetes: the A therosclerosis R isk in C ommunities S tudy. Diabetes, Obesity and Metabolism. 2016;18(9):899-906.

31. Levey AS, Stevens LA, Schmid CH, Zhang YL, Castro AF, Feldman HI, et al. A new equation to estimate glomerular filtration rate. Annals of internal medicine. 2009;150(9):604-12.

32. Rubin DB. Multiple imputation for nonresponse in surveys: John Wiley \& Sons; 2004.

33. Harrell Jr FE, Lee KL, Califf RM, Pryor DB, Rosati RA. Regression modelling strategies for improved prognostic prediction. Statistics in medicine. 1984;3(2):143-52.

34. Billah B, Huq MM, Smith JA, Sufi F, Tran L, Shardey GC, et al. AusSCORE II in predicting 30-day mortality after isolated coronary artery bypass grafting in Australia and New Zealand. The Journal of thoracic and cardiovascular surgery. 2014;148(5):1850-5. e2.

35. Javanbakht M, Abolhasani F, Mashayekhi A, Baradaran HR. Health related quality of life in patients with type 2 diabetes mellitus in Iran: a national survey. PloS one. 2012;7(8):e44526.

36. Solli $\mathrm{O}$, Stavem K, Kristiansen IS. Health-related quality of life in diabetes: The associations of complications with EQ-5D scores. Health and quality of life outcomes. 2010;8(1):18.

37. Sakamaki H, Ikeda S, Ikegami N, Uchigata Y, Iwamoto Y, Origasa $H$, et al. Measurement of HRQL using EQ-5D in patients with type 2 diabetes mellitus in Japan. Value in Health. 2006;9(1):47-53.

38. Lee WJ, Song K-H, Noh JH, Choi YJ, Jo M-W. Health-related quality of life using the EuroQol 5D questionnaire in Korean patients with type 2 diabetes. Journal of Korean medical science. 2012;27(3):255-60.

39. O’Reilly DJ, Xie F, Pullenayegum E, Gerstein HC, Greb J, Blackhouse GK, et al. Estimation of the impact of diabetes-related complications on health utilities for patients with type 2 diabetes in Ontario, Canada. Quality of Life Research. 2011;20(6):939-43.

40. Takahara M, Katakami N, Shiraiwa T, Abe K, Ayame H, Ishimaru Y, et al. Evaluation of health utility values for diabetic complications, treatment regimens, glycemic control and other subjective symptoms in diabetic patients using the EQ-5D-5L. Acta Diabetologica. 2019;56(3):309-19.

41. Lu Y, Wang N, Chen Y, Nie X, Li Q, Han B, et al. Health-related quality of life in type-2 diabetes patients: a cross-sectional study in East China. BMC endocrine disorders. 2017;17(1):38.

42. Mahmoud SS, Mahdy MHE, Mahfouz MS, Nada IS, Aqeeli AA, Darbi MAA, et al. Effects of a Psychoeducational Program on Hemoglobin A1c Level and Health-Related Quality of Life in Patients with Type 2 Diabetes Mellitus, Jazan, Saudi Arabia. BioMed research international. $2018 ; 2018$.

43. Pucci GCMF, Rech CR, Fermino RC, Reis RS. Association between physical activity and quality of life in adults. Revista de Saúde Pública. 2012;46(1):16679.

44. Anokye NK, Trueman P, Green C, Pavey TG, Taylor RS. Physical activity and health related quality of life. BMC public health. 2012;12(1):624.

45. Brown AF, Ettner SL, Piette J, Weinberger M, Gregg E, Shapiro MF, et al. Socioeconomic position and health among persons with diabetes mellitus: a conceptual framework and review of the literature. Epidemiologic reviews. 2004;26(1):63-77.

46. Kurpas D, Mroczek B, Bielska D. Rural and urban disparities in quality of life and health-related behaviors among chronically ill patients. Rural Remote Health. 2014;14:2485.

47. Derakhshanpour F, Vakili MA, Farsinia M, Mirkarimi K. Depression and quality of life in patients with type 2 diabetes. Iranian Red Crescent Medical Journal. 2015;17(5).

48. Paschalides C, Wearden A, Dunkerley R, Bundy C, Davies R, Dickens C. The associations of anxiety, depression and personal illness representations with glycaemic control and health-related quality of life in patients with type 2 diabetes mellitus. Journal of psychosomatic research. 2004;57(6):557-64.

49. Snoek FJ, Skinner TC. Psychology in diabetes care: John Wiley \& Sons; 2007.

50. American Diabetes Association. Quality of life in type 2 diabetic patients is affected by complications but not by intensive policies to improve blood glucose or blood pressure control (UKPDS 37). UK Prospective Diabetes Study Group. Diabetes care. 1999;22(7):1125-36. 